\title{
Increasing the interfacial strength in carbon fiber/polypropylene composites by growing CNTs on the fibers
}

\author{
S. Yumitori ${ }^{1}$, Y. Arao ${ }^{2}$, T. Tanaka ${ }^{2}$, K. Naito ${ }^{3}$, K. Tanaka ${ }^{4}$ \\ \& T. Katayama ${ }^{4}$ \\ ${ }^{1}$ New Energy and Industrial Technology Development Organization, \\ Japan \\ ${ }^{2}$ Department of Mechanical and Systems Engineering, \\ Doshisha University, Japan \\ ${ }^{3}$ National Institute for Materials Science, Japan \\ ${ }^{4}$ Department of Biomedical Engineering, Japan
}

\begin{abstract}
Carbon nanotubes (CNTs) were grown uniformity on the surface of carbon fibers to create hierarchical fibers by use of floating catalyst chemical vapor deposition. The tensile properties of CNTs grafted fibers were measured. The strength of grafted fibers decreased approximately 12\% compared to raw carbon fibers in this process. A fiber pull-out test revealed that the interfacial shear strength (IFSS) in polypropylene composites improved by 35\% by grafting CNTs onto carbon fibers. It indicates the use of hierarchical fibers in thermoplastic composites is effective due to the improvement of IFSS by mechanical interlocking between CNTs and the matrix. The CNT/fiber joint strength is the most critical property, and the experimental observations also revealed that the joint fracture was the major failure mode.
\end{abstract}

Keywords: hierarchical reinforcement, carbon nanotube, carbon fibers, interface, thermoplastics.

\section{Introduction}

Carbon fibers have been widely used for advanced structural composites in various areas such as airplanes, automotive, and sporting goods because of their 
high specific strength and modulus. The performance of carbon-fiber reinforced plastics was mainly determined by fiber volume fraction, fiber length, and fibermatrix interface. With respect to mass production such as automotive industry, short-fiber reinforced thermoplastics (SFRTP) have been adopted due to their processability and renewability [1-4]. Glass fiber/polypropylene composite system is usually used in the automotive parts. However, this material system cannot be used as primary structures due to its low stiffness and strength. Adopting carbon fiber instead of glass fiber improves the stiffness of the composites to some extent, but the strength of composites is not modified because of low IFSS strength between carbon fiber and polypropylene. Leaving long fiber during manufacturing process and modifying IFSS using compatibilizer are the solutions to improve the strength of SFRP [5, 6]. Drastic strength and stiffness improvement is required for SFRP to adopt them in primary structures

The huge interest in CNTs has been simulated by their extraordinary intrinsic properties. The introduction of CNTs into conventional fibre reinforced polymer composites creates a hierarchical reinforcement structure and can improve composites performance [7-17]. The addition of CNTs into conventional composites modifies the IFSS of composites; therefore it can improve the strength of them. However, there are difficulties of dispersing and aligning high loading fractions of CNTs. Grafting CNTs onto fibre surface is an effective method to solve above problems. In addition, grafting CNT can improve IFSS by introducing mechanical interlocking, and strength in the transverse direction of fibers that are weak point of FRPs.

Thostenson et al. [7] first successfully synthesized CNT via thermal chemical vapor deposition (CVD) on the surface of carbon fiber, using a pre-deposited metal catalyst. The morphology of CNT can be controlled by changing temperature, catalyst, hydrocarbon source and processing time [9, 12]. The types of catalysis affect the most, and adhesion energy and diffusion rates of catalyst on the fiber surface are important parameters. IFSS improvement for epoxy/grafted carbon fiber systems have been reported by many authors [8-13]. CNTs on fiber surfaces show capillary action to epoxy resin. IFSS improvement reported by other researchers is in the range of $20-470 \%$, depends on the CNT growing methods.

Recently, the effects of hierarchical fiber on the strength of SFRP were reported. Vishkaei et al. [14] and Rahmanian et al. [15] confirmed approximately $40 \%$ and $30 \%$ enhancement in tensile strength and stiffness compared to conventional SFRP, respectively. They concluded that in addition to causing enhanced fiber-matrix interfaced bonding, CNT also act as reinforcement. The viscosity of thermoplastics is higher than that of epoxy resin. Therefore, the capillary action may not occur in the case of using thermoplastics. The effect of CNT grafting on the improvement of IFSS between fiber and thermoplastics is not clear.

In this study, CNTs were grafted onto a carbon fiber surface by floating CVD method. IFSS between fiber and polypropylene was evaluated by fiber pull-out test. Fiber surface after pull-out test was observed to discuss the interfacial 
failure mechanism for grafted fiber. In addition, strength of fiber before and after grafting CNT was investigated.

\section{Experimental}

\subsection{Catalytic growth of CNTs on carbon fiber}

The carbon fiber used in this study was PAN-based (T300) carbon fiber. The T300 fiber was supplied from Toray Industries, Inc. Carbon fiber in the asreceived state had been subjected to commercial surface treatment and sizing (epoxy compatible sizing).

The growth of CNTs on carbon fibers was performed by floated CVD in a reactor furnace. The sizing of carbon fiber bundle was removed by heating at $750^{\circ} \mathrm{C}$ on $\mathrm{SiC}$ heater for an hour in vacuum. The $\mathrm{Fe}\left(\mathrm{C}_{2} \mathrm{H}_{5}\right)_{2}$ (ferrocene) catalyst was placed under the fiber and evaporated to deposit it onto carbon fiber surface. Then, ethanol as a carbon source was injected. The growth temperature and time for CNT deposition were selected as $750^{\circ} \mathrm{C}$ for $900 \mathrm{~s}$. The CNTs grown on carbon fibers were examined using a high resolution scanning electron microscope (SEM) (S-4500, Hitachi).

\subsection{Single fiber tensile test}

In order to determine the effect of the CNT growth on the axial properties, the tensile strength of each fiber was measured based on JIS R 7606 (Japanese Industrial Standard). A single carbon fiber was glued at the either end onto a paper frame with epoxy. The diameter of single carbon fiber was measured by SEM prior to the tests. The average diameter of the fiber was $7.4 \mu \mathrm{m}$. It is difficult to determine the diameter of grafted fiber. Therefore, the average diameter of as-received carbon fiber was used as diameter of grafted fibers. Tensile tests of single carbon fibers were performed using micro-servo testing instrument (MMT-11N, Shimadzu Co.) with a cross-head speed of $1 \mathrm{~mm} / \mathrm{min}$. More than 10 single fibers were used to determine the tensile strength at gauge length of $20 \mathrm{~mm}$.

\subsection{Fiber pull-out test}

In order to obtain a value of IFSS, fiber pull-out tests were conducted. Figure 1 shows the schematic representation of fiber pull-out test. The polypropylene used in this study is low viscous type (J108M, Prime Polymer Co., Ltd.) Melt flow index was $45 \mathrm{~g} / 10 \mathrm{~min}$. Three types of carbon fibers (as-received, acetone cleaned fiber and grafted carbon fiber) were prepared. The polypropylene was melted on a hotplate at $180^{\circ} \mathrm{C}$. Then, carbon fibers were embedded into the molten resin using a manipulator. After the resin was cooled to room temperature, the fiber pull-out test was conducted at a crosshead speed of $0.1 \mathrm{~mm} / \mathrm{min}$. The load-displacement curve for each sample was recorded in each test and the peak force $F_{\max }$ was obtained. After the fiber pull-out test, SEM observations were performed to determine the embedded length $l_{\mathrm{e}}$ and diameter $d$ 
of the fiber. The embedded fiber lengths varied in a range from 10 to $500 \mu \mathrm{m}$. IFSS was calculated using the equation

$$
\tau=\frac{F_{\max }}{\pi d l_{e}}
$$

Over five tests were conducted to obtain the average IFSS.

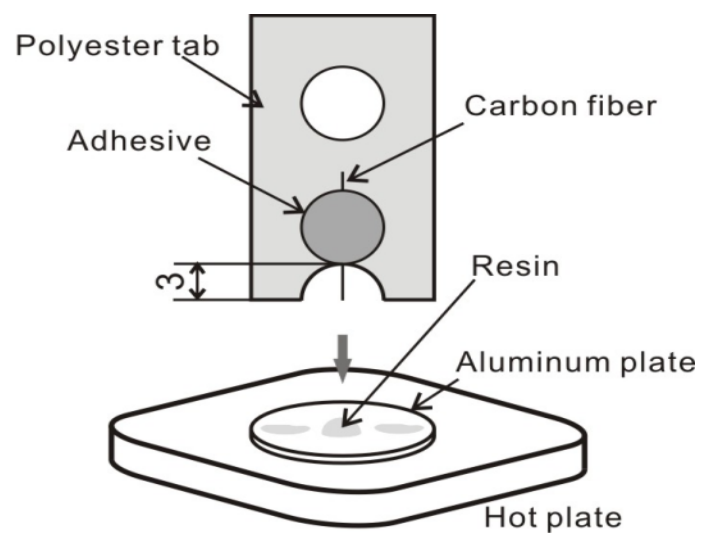

Figure 1: $\quad$ Schematic representation of the fiber pull-out test.

\section{Results and discussion}

\subsection{Surface morphology of grafted fiber}

Figure 2 shows the surface morphology of as-received (epoxy sized), acetone cleaned fiber and CNT grafted fiber. The surface of carbon fiber did not show any changes after acetone cleaning. T-300 fiber has groove-like features parallel to the fiber axis, and the surface of fibers is not smooth. By using these not smooth fibers as substrate of CNTs, randomly oriented CNTs were obtained on carbon fiber surface as shown in Fig. 2(c). The CNT grown on the fibers are multi-walled CNTs (MECNTs) and the diameters of CNTs are in the range of 20-60 nm. The apparent diameter of grafted carbon fiber increased by $5-10 \%$ by the catalytic growth of CNTs. CNTs were easily peeled from carbon fiber. It indicated the CNT/fiber joint strength is not strong. In this experiment, entangled CNTs were obtained on the carbon fiber. Aligned CNTs can be obtained if we use smooth carbon fiber and choose proper catalysis and deposit method [12].

\subsection{Single fiber tensile tests}

Figure 3 and Table 1 show the results of single fiber tensile results. The acetone cleaning did not affect the strength of carbon fiber. On the other hand, grafting CNT onto fiber decreased the strength of fibers approximately $9 \%$. Note that we 


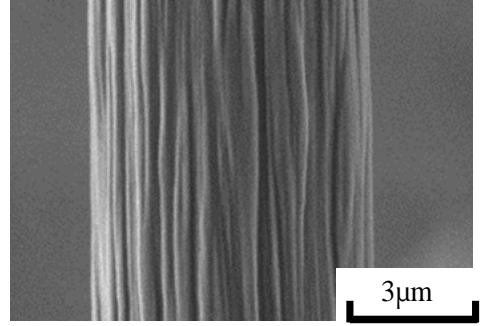

(a) As-received fiber

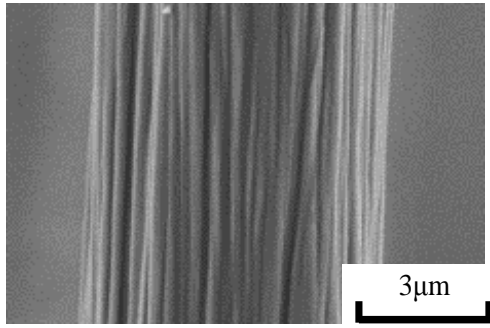

(b) Acetone cleaned fiber

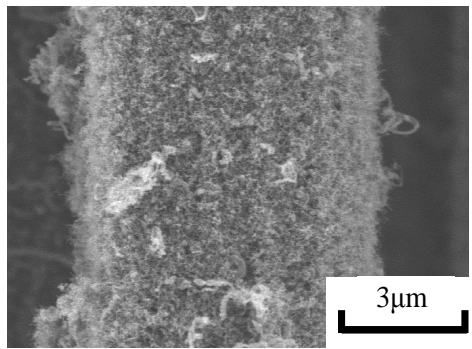

(c) CNT grafted fiber

Figure 2: $\quad$ Surface morphology of carbon fiber and CNT grafted fiber.

did not consider the increased diameter of grafting fiber. It means the strength of carbon fiber itself decreased after grafting process. Strength reduction behaviour during grafting process corresponds to other research which reported a reduction in tensile strength of carbon fibers as much as 10-50\% [8, 11, 12]. Qian et al. [8] observed carbon fiber surface after CNTs grafting by removing catalyst and CNTs using hydrochloric acid to investigate the strength reduction of grafting fibers. They reported that lots of nano-size pits, which were formed by dissolution of Fe ion generated by ferrocene, were observed. The defect generated by catalyst depositing process reduces the strength of carbon fiber. As opposed to the above results, Naito et al. [16] and Kim et al. [17] reported that the strength of carbon fibers increased by more than $10 \%$ during CVD process. Kim et al. [17] found that the repair to some of the damage incurred during the catalytic deposition, an increase of carbon crystal size, and the formation of crosslinks of neighboring crystal all occur during CVD process, and contribute the improvement. In this study, such healing mechanism did not occur, therefore the strength of grafted fiber decreased by the pits generated by catalytic deposition.

Table 1: Weibull parameters.

\begin{tabular}{cccc}
\hline & As-received fiber & Cleaned fiber & CNT grafted fiber \\
\hline$m$ & 7.9 & 9.3 & 4.8 \\
$\sigma_{0} \mathrm{MPa}$ & 3120 & 3030 & 2680 \\
\hline
\end{tabular}




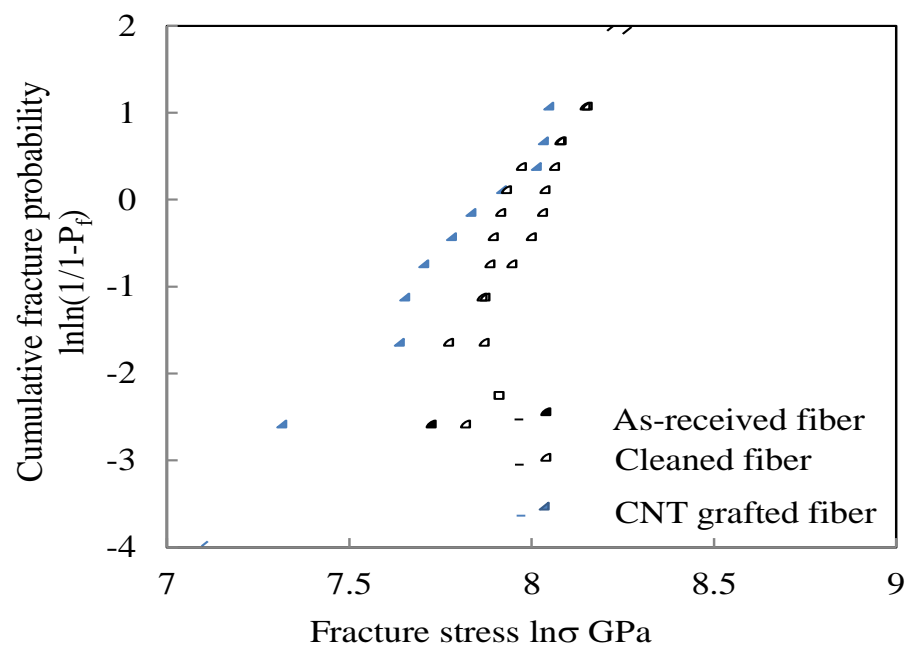

Figure 3: $\quad$ Weibull distribution of single fiber tensile test.

\subsection{Fiber pull-out tests}

Figure 4 shows the relationships between fibers embedded length in polypropylene and maximum load in the fiber pull-out tests. IFSS determined by the test is also shown in Fig. 4. The IFSS for carbon fiber/polypropylene was approximately 8.8 MPa. IFSS of this material systems slightly increased by removing epoxy sizing. In either case, IFSS for carbon fiber/polypropylene system is smaller compared to that based on epoxy resin (more than $15 \mathrm{MPa}$ ) [11-13]. Carbon fiber exhibit poor interactions with most polymers owing to their nonpolar surface. In addition, because PP is a nonpolar polymer, the wettability of and adhesion to the fiber are smaller compared to other polymer.

IFSS improved by $35 \%$ by grafting CNT onto the fiber surface compared to IFSS of cleaned fiber. It is confirmed that the CNT grafting onto carbon fiber is effective to modify IFSS of carbon fiber/polypropylene systems, though the penetration and wettability of polypropylene to grafted fibers were concerned. In this study, the thickness of CNT layer was less than $1 \mu \mathrm{m}$, and thinner compared to other researches $[12,15]$. In the case of thin CNT layer, polypropylene can penetrate between CNT, and thus improve IFSS by mechanical interlocking effect of CNT. To investigate the fracture mechanism during fiber pull-out test, the fiber surface after the test was observed as shown in Fig. 5. By comparing with Fig. 2(c) and Fig. 5, we can see the penetration of polypropylene into CNT layer. In addition, bare carbon fiber surface was observed after the tests. It indicates that the fracture occurred at fiber/CNT joint. In fact, the fiber/CNT joint strength is weak, and CNT was easily detached from fiber surface when we abstracted single fiber from fiber bundle. Fig. 6 shows the possible fracture modes for CNT grafted fiber. If the fiber/CNT joint strength is weakest, joint 
fracture is observed as shown in Fig. 6(a), and this fracture mode is observed in this experiment. Fracture mode as shown in Fig. 6(b) and (c) is preferable to strengthen CNT's role. In these modes, IFSS of grafted fiber will be further improved. The joint strength of fiber and CNT can be controlled by grafting method. Kim et al. successfully created grafted fiber with high joint and fiber strength [13]. In their experiment, $\mathrm{Ni}$ as catalyst of CNT was diffused into carbon fibers by annealing process. They observed that the root of the CNT has been immersed into the graphic layers below the carbon fiber surface as a result of the embedded Ni particles inside the carbon fibers. In this case, about $470 \%$ of IFSS improvement was observed. In our floating CVD process, diffusion and annealing of catalyst did not conduct; hence the joint strength is not enough to restrain joint fracture during fiber pullout. The fiber/CNT joint strength is the key factor to improve IFSS between grafted fiber and polypropylene.

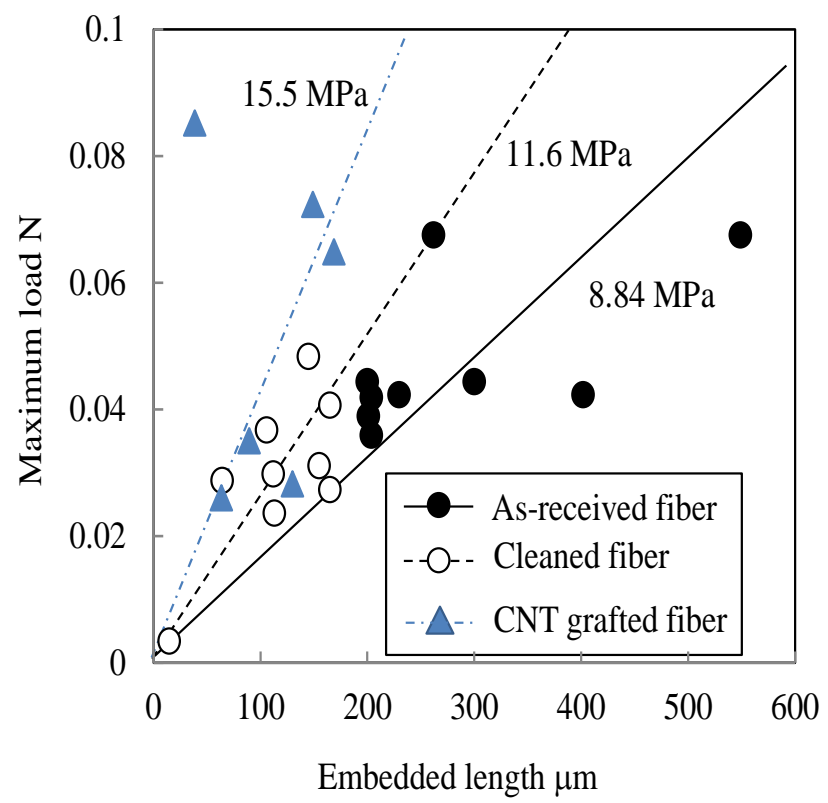

Figure 4: Relationships between embedded fiber length and maximum load. 


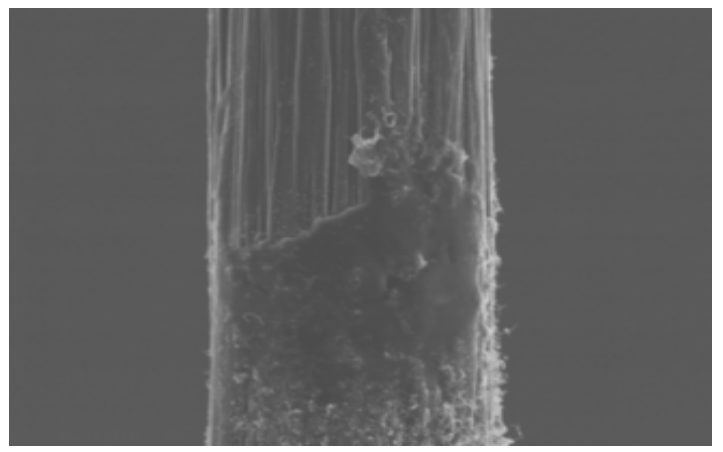

Figure 5: $\quad$ Surface of CNT grafted fiber after pull- out test.

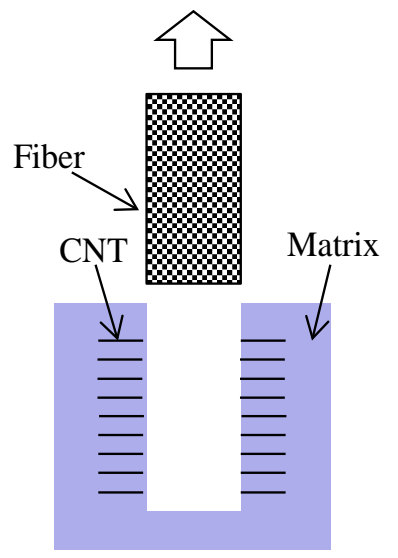

(a) CNT/fiber joint fracture

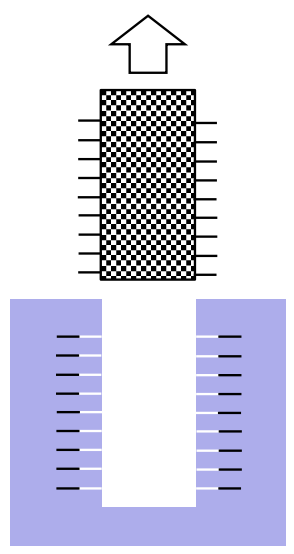

(b) CNT fracture

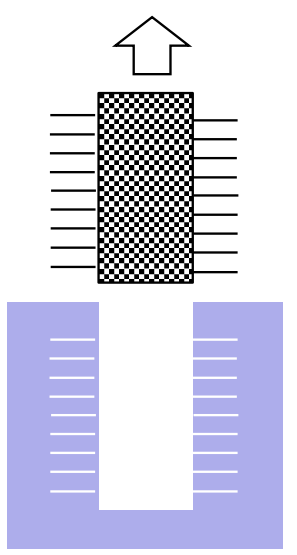

(c) Pull out of CNT

Figure 6: Fracture types of grafted carbon fiber during fiber pullout.

\section{Conclusions}

CNTs were grown onto the carbon fiber to improve the IFSS for composites based on polypropylene. CNTs were successfully grafted on fiber surface by floated CVD method. Entangle CNT layer with the thickness of less than $1 \mu \mathrm{m}$ was obtained. The strength of grafted fiber was decreased by $12 \%$ due to the defects generated by catalyst depositing process. Fiber pull-out tests were conducted to evaluate IFSS of the composites. Polypropylene was penetrated into the CNTs, and the IFSS was increased by 35\% by grafting CNT onto the fiber. The fiber/CNT joint fracture was observed after pull-out test. The weak joint strength deterrents the IFSS improvement for grafted fibers. 


\section{References}

[1] Rezaei F, Yunus R, Ibrahim NA, Mahdi ES. Development of short-carbonfiber-reinforced polypropylene composite for car bonnet. Polymer-Plastics Technology and Engineering 2008; 47: 351-357.

[2] Thomason JL. Influence of fibre length and concentration on the properties of glass fibre-reinforced polypropylene: 5. Injection moulded long and short fibre PP. Composite Part A 2002; 33: 1641-1652.

[3] Fu SY, Lauke B, Mäder E, Yue CY, Hu X. Tensile properties of shortglass- and short-carbon-fiber-reinforced polypropylene composites. Composite Part A 2000; 31: 1117-1125.

[4] Kumar MS, Ghosh AK, Bhatnagar N. Mechanical properties of injection molded long fiber polypropylene composite, Part 1: Tensile and flexural properties. Polym Compos 2007; 28: 259-266.

[5] Fu X, He B, Chen X. Effects of compatibilizers on mechanical properties of long glass fiber-reinforces polypropylene. J Reinf Plast Compos 2010; 29: 936-949.

[6] Karsli NG, Aytac A. Effects of maleated polypropylene on the morphology, thermal and mechanical properties of short carbon fiber reinforced polypropylene composites. Mater Design 2011; 32: 4069-4073.

[7] Thostenson ET, Li WZ, Wang DZ, Ren ZF, Chou TW, Carbon nanotube/carbon fiber hybrid multiscale composites, J Appl Phys 2002; 91: 6034-6037.

[8] Qian H, Bismarck A, Greenhalgh ES, Kalinka G, Shaffer MSP. Hierarchical composites reinforced with carbon nanotube grafted fibers: The potential assessed at the single fiber level. Chem Mater 2008; 20: 1862-1869.

[9] Qian H, Greenhalgh ES, Shaffer MSP, Bismarck A. Carbon nanotube-based hierarchical composites: a review, J Mater Chem 2010; 20: 4751-4762.

[10] Kamae T, Drzal LT. Carbon fiber/epoxy composite property enhancement through incorporation of carbon nanotubes at the fiber-matrix interphasePart I:The development of carbon nanotube coated carbon fibers and the evaluation of their adhesion. Compos A 2012; 43: 1569-1577.

[11] Qian H, Bismarck A, Greenhalgh ES, Shaffer MSP. Carbon nanotube grafted carbon fiber: A study of wetting and fibre fragmentation. Compos A 2010; 41: 1107-1114.

[12] Lv P, Feng Y, Zhang P, Chen H, Zhao N, Feng W. Increasing the interfacial strength in carbon fiber/epoxy composites by controlling the orientation and length of carbon nanotubes grown on the fibers, Carbon 2011; 49: 4665-4673.

[13] Kim KJ, Kim J, Yu W, Youk JH, Lee J. Improved tensile strength of carbon fibers undergoing catalytic growth of carbon nanotubes on their surface. Carbon 2013; 54: 258-267.

[14] Vishkaei MS, Salleh MAM, Yunus R, Biak DRA, Danafar F, Mirjalili F. Effect of short carbon fiber surface treatment on composite properties. J Compos Mater 2010; 45: 1885-1891. 
[15] Rahmanian S, Thean KS, Suraya AR, Shazed MA, Salleh MAM, Yusoff HM. Carbon and glass hierarchical fibers: Influence of carbon nanotubes on tensile, flexural and impact properties of short fiber reinforced composites. Mater Design 2013; 43: 10-16.

[16] Naito K, Yang J, Tanaka Y, Kagawa Y. Tensile properties of carbon nanotubes grown on ultrahigh strength polyacrylonitrile-based and ultrahigh modulus pitch-based carbon fibers. Appl Phy Lett 2008; 231912.

[17] Kim KJ, Yu W, Youk JH, Lee J. Degradation and healing mechanisms of carbon fibers during the catalytic growth of carbon nanotubes on their surfaces. Appl Mater Interfaces 2012; 4: 2250-2258. 